\title{
EXCISION IN HOPF CYCLIC HOMOLOGY
}

\author{
ATABEY KAYGUN AND MASOUD KHALKHALI
}

\section{INTRODUCTION}

Cyclic cohomology theory for Hopf algebras was introduced by Connes and Moscovici in their ground breaking paper on transverse index theory [3] (cf. also [4 [5], and [6] for a recent survey and new results). This theory can be regarded as the right noncommutative analogue of Lie algebra homology because of existence of a characteristic map for actions of Hopf algebras and since it reduces to Lie algebra homology for Hopf algebras associated to Lie algebras. This should be compared with the role played by ordinary cyclic cohomology of noncommutative algebras and its reduction to de Rham homology for smooth commutative algebras [2]. The algebraic underpinnings of Hopf cyclic theory however turned out to be more complicated. It is now understood as a special case of a general theory developed in the series of papers 13, 14, 10, 11] (with the help of [1]) for (co)algebras endowed with a (co)action of a Hopf algebra and where, among other things, the right notion of coefficients was introduced (cf. also [15] for a recent survey). This theory was later extended by the first author [12] to bialgebras and to a much larger class of coefficients. This extension is rather surprising since existence of a bijective antipode was essential in constructing the cyclic and cocyclic complexes developed for Hopf cyclic homology of both variants. By bialgebra or Hopf cyclic homology we now mean the theory expounded in these papers mentioned above.

The main results of the present paper are two excision theorems (Theorem 4.11] and Theorem [7.7below) for Hopf cyclic homology of module coalgebras and comodule algebras respectively. A basic property of cyclic (co)homology for algebras and coalgebras is excision. If by cyclic theory we mean the positively graded cyclic theory then this question is completely settled by Wodzicki's excision theorem [17. Originally formulated for algebras, this result states that a not necessarily unital $k$-algebra $I$ satisfies the excision property in cyclic homology for all algebra extensions $0 \rightarrow I \rightarrow A \rightarrow A / I \rightarrow 0$ if and only if $I$ is $H$ unital (cf. 9] for the corresponding coalgebra result). It is this result that we generalize in this paper by finding sufficient conditions for excision to hold in Hopf cyclic cohomology. It is known after Cuntz and Quillen [7] that for periodic cyclic cohomology excision always holds irrespective of $I$ being $H$-unital or not. Wodzicki's theorem is used in the proof of this result and we believe that the corresponding statements hold for periodic Hopf cyclic homology as well and we state two conjectures to this effect (Conjecture 4.14 and Conjecture 7.10 below). 
Throughout this paper we will work over a field $k$ of an arbitrary characteristic. One can prove all results we prove in this paper by requiring all $k$-modules we use to be $k$-projective or more generally $k$-flat, if one needs $k$ to be just a commutative algebra with a unit. Unadorned tensor products $\otimes$ are over $k$ and all other tensor products will be clearly indicated. All algebras (resp. coalgebras) will be associative (resp. coassociative) but not necessarily unital (resp. counital). The comultiplication and counit of a coalgebra $C$ will be denoted by $\Delta$ and $\varepsilon$, respectively. The unit of an algebra $A$ will be denoted by $\mathbb{I}$. $B$ will always be a unital and counital, associative and coassociative bialgebra, or a Hopf algebra. All modules over $B$ are assumed to be left modules unless it is stated otherwise. For a $B$-module $X$ we will distinguish an element $b \in B$ and the $k$-module endomorphism $L_{b}$ of $X$ defined by the left action of $b$ on $X$. If $X$ is simultaneously a $B$-module and a $B$-comodule with no compatibility between these structures assumed, we will call $X$ a $B$-module/comodule. We will use Sweedler's notation denoting comultiplication structure on coalgebras by $\Delta(c)=c_{(1)} \otimes c_{(2)}$. Similarly if $X$ is a left (resp. right) comodule over a coalgebra $C$ with coaction $X \stackrel{\rho_{X}}{\longrightarrow} C \otimes X$ (resp. $X \stackrel{\rho_{X}}{\longrightarrow} X \otimes C$ ), we write $\rho_{X}(x)=x_{(-1)} \otimes x_{(0)}\left(\right.$ resp. $\left.\rho_{X}(x)=x_{(0)} \otimes x_{(1)}\right)$ to denote its structure map where a summation is understood.

\section{Module COALGEBRAS AND EQUivariant COMODUleS}

Let $B$ be a bialgebra. Recall that a coalgebra $C$ is called a left $B$-module coalgebra if $C$ is a left $B$-module and its comultiplication $C \stackrel{\Delta}{\longrightarrow} C \otimes C$ is a $B$-module morphism. Explicitly

$$
(b(c))_{(1)} \otimes(b(c))_{(2)}=b_{(1)}\left(c_{(1)}\right) \otimes b_{(2)}\left(c_{(2)}\right)
$$

for any $c \in C$ and $b \in B$.

Let $C$ be a coalgebra, $X \stackrel{\rho_{X}}{\longrightarrow} X \otimes C$ be a right $C$-comodule and $Y \stackrel{\rho_{Y}}{\longrightarrow} C \otimes Y$ be a left $C$-comodule. Recall that the cotensor product $X \underset{C}{\square} Y$ is defined as the kernel

$$
X \underset{C}{\square} Y:=\operatorname{ker}\left(X \otimes Y \stackrel{\left(\rho_{X} \otimes i d_{Y}\right)-\left(i d_{X} \otimes \rho_{Y}\right)}{\longrightarrow} X \otimes C \otimes Y\right)
$$

Explicitly, $X \underset{C}{\square} Y$ is the $k$-submodule of $X \otimes Y$ generated over $k$ by terms of the form $\sum_{i}\left(x^{i} \otimes y^{i}\right)$ which satisfy

$$
\sum_{i} x_{(0)}^{i} \otimes x_{(1)}^{i} \otimes y=\sum_{i} x^{i} \otimes y_{(-1)}^{i} \otimes y_{(0)}^{i}
$$

The derived functors of the cotensor product are denoted by $\operatorname{Cotor}_{*}^{C}(\cdot, \cdot)$.

Definition 2.1. Let $C$ be a left $B$-module coalgebra. A $k$-module $M$ is called a $B$-equivariant left $C$ comodule if: (i) $M$ is a left $B$-module, (ii) $M$ is a left $C$-comodule, and (iii) the $C$-coaction $M \stackrel{\rho_{M}}{\longrightarrow} C \otimes M$ 
is a $B$-module morphism, in other words

$$
(b(m))_{(-1)} \otimes(b(m))_{(0)}=b_{(1)}\left(m_{(-1)}\right) \otimes b_{(2)}\left(m_{(0)}\right)
$$

for all $b \in B$ and $m \in M$.

Definition 2.2. Let $C$ be a coalgebra. We define a differential graded $k$-module $\mathrm{CB}_{*}^{\text {bar }}(C)$ by letting $\mathrm{CB}_{n}^{\mathrm{bar}}(C)=C^{\otimes n+2}$ and defining a pre-simplicial structure

$$
\partial_{j}\left(c^{0} \otimes \cdots \otimes c^{n+1}\right)=c^{0} \otimes \cdots \otimes c_{(1)}^{j} \otimes c_{(2)}^{j} \otimes \cdots \otimes c^{n+1}
$$

for any $n \geq 0,0 \leq j \leq n+1$ and $\left(c^{0} \otimes \cdots \otimes c^{n+1}\right)$ from $\mathrm{CB}_{n}^{\mathrm{bar}}(C)$. The corresponding differential is defined as $d_{n}^{\mathrm{CB}}=\sum_{j=0}^{n+1}(-1)^{j} \partial_{j}$. The differential graded $k-$ module $\mathrm{CB}_{*}^{\mathrm{bar}}(C)$ is called the bar resolution of $C$. Note that $\mathrm{CB}_{*}^{\mathrm{bar}}(C)$ need not be a resolution for a non-counital coalgebra $C$.

Proposition 2.3. Let $C$ be a left $B$-module coalgebra. Then the bar resolution $\mathrm{CB}_{*}^{\text {bar }}(C)$ is a differential graded B-equivariant C-bicomodule.

Proof. The left and right $C$-comodule structures are defined as

$$
\begin{aligned}
& \rho_{L}\left(c^{0} \otimes \cdots \otimes c^{n+1}\right)=c_{(1)}^{0} \otimes\left(c_{(2)}^{0} \otimes \cdots \otimes c^{n+1}\right) \\
& \rho_{R}\left(c^{0} \otimes \cdots \otimes c^{n+1}\right)=\left(c^{0} \otimes \cdots \otimes c_{(1)}^{n+1}\right) \otimes c_{(2)}^{n+1}
\end{aligned}
$$

and the $B$-module structure is diagonal

$$
L_{b}\left(c^{0} \otimes \cdots \otimes c^{n+1}\right)=b_{(1)}\left(c^{0}\right) \otimes \cdots \otimes b_{(n+1)}\left(c^{n+1}\right)
$$

defined for any $b \in B, n \geq 0$ and $c^{0} \otimes \cdots \otimes c^{n+1}$ from $\mathrm{CB}_{n}^{\mathrm{bar}}(C)$. As for the $C$-coaction being $B$-equivariant we check

$$
\begin{aligned}
L_{b} \rho_{L}\left(c^{0} \otimes \cdots \otimes c^{n+1}\right) & =b_{(1)}\left(c_{(1)}^{0}\right) \otimes b_{(2)}\left(c_{(2)}^{0}\right) \otimes b_{(3)}\left(c^{1}\right) \otimes \cdots \otimes b_{(n+3)}\left(c^{n+1}\right) \\
& =b_{(1)}\left(c_{(1)}^{0}\right) \otimes L_{b_{(2)}}\left(c_{(2)}^{0} \otimes \cdots \otimes c^{n+1}\right) \\
& =\rho_{L} L_{b}\left(c^{0} \otimes \cdots \otimes c^{n+1}\right)
\end{aligned}
$$

for any $b \in B, n \geq 0$ and $\left(c^{0} \otimes \cdots \otimes c^{n+1}\right)$ from $\mathrm{CB}_{n}^{\mathrm{bar}}(C)$. The proof for the right coaction is similar. So far we only showed that $\mathrm{CB}_{*}^{\text {bar }}(C)$ is a graded $B$-equivariant $C$-bicomodule. We must show that the pre-simplicial structure is compatible with the $C$-bicomodule structure and the $B$-module structures. We 
start with the $B$-module structure

$$
\begin{aligned}
& L_{b} \partial_{j}\left(c^{0} \otimes \cdots \otimes c^{n+1}\right) \\
& \quad= \begin{cases}b_{(1)}\left(c_{(1)}^{0}\right) \otimes b_{(2)}\left(c_{(2)}^{0}\right) \otimes b_{(3)}\left(c^{1}\right) \otimes \cdots \otimes b_{(n+3)}\left(c^{n+1}\right) & \text { if } j=0 \\
b_{(1)}\left(c^{0}\right) \otimes \cdots \otimes b_{(j+1)}\left(c_{(1)}^{j}\right) \otimes b_{(j+2)}\left(c_{(2)}^{j}\right) \otimes \cdots \otimes b_{(n+3)}\left(c^{n+1}\right) & \text { if } 1 \leq j \leq n \\
b_{(1)}\left(c^{0}\right) \otimes \cdots \otimes b_{(n+1)}\left(c^{n}\right) \otimes b_{(n+2)}\left(c_{(1)}^{n+1}\right) \otimes b_{(n+3)}\left(c_{(2)}^{n+1}\right) & \text { if } j=n+1\end{cases} \\
& =\partial_{j} L_{b}\left(c^{0} \otimes \cdots \otimes c^{n+1}\right)
\end{aligned}
$$

As for the $C$-comodule structure

$$
\begin{aligned}
\left(i d_{C} \otimes \partial_{j}\right) \rho_{L}\left(c^{0} \otimes \cdots \otimes c^{n+1}\right) & = \begin{cases}c_{(1)}^{0} \otimes c_{(2)}^{0} \otimes c_{(3)}^{0} \otimes c^{1} \otimes \cdots \otimes c^{n+1} & \text { if } j=0 \\
c_{(1)}^{0} \otimes c_{(2)}^{0} \otimes \cdots \otimes c_{(1)}^{j} \otimes c_{(2)}^{j} \otimes \cdots & \text { if } 1 \leq j \leq n+1\end{cases} \\
& =\rho_{L} \partial_{j}\left(c^{0} \otimes \cdots \otimes c^{n+1}\right)
\end{aligned}
$$

The proof for the right coaction is similar.

Lemma 2.4. Let $C$ be a $B$-module coalgebra, $X$ be a $B$-equivariant right $C$-comodule and $Y$ be a $B$ equivariant left $C$-comodule. Then $X \underset{C}{\square} Y$ is a B-module.

Proof. The left $B$ action on $X \otimes Y$ is diagonal. We check that $X \square_{C} Y$ is a $B$-submodule. Take $\Psi$ from $X \underset{C}{\square} Y$ and $b \in B$. Then

$$
\left(\rho_{X} \otimes i d_{Y}\right) L_{b}(\Psi)=L_{b}\left(\rho_{X} \otimes i d_{Y}\right)(\Psi)=L_{b}\left(i d_{X} \otimes \rho_{Y}\right)(\Psi)=\left(i d_{X} \otimes \rho_{Y}\right) L_{b}(\Psi)
$$

proving what we wanted.

Definition 2.5. An object $X$ in the category of right (resp. left) $C$-comodules is called co-projective if the functor $\operatorname{Hom}_{\text {Comod-C}}(X, \cdot)$ (resp. $\operatorname{Hom}_{C-\text { Comod }}(X, \cdot)$ ) is an exact functor from the category of right (resp. left) $C$-comodules into the category of $k$-modules.

Proposition 2.6. Let $C$ be a counital B-module coalgebra and $X$ and $Y$ be as before. Then the cotorsion groups $\operatorname{Cotor}_{*}^{C}(X, Y)$ are canonically B-modules.

Proof. The existence of right derived functors of the functors $X \underset{C}{\square} \cdot$ and $\cdot{ }_{C} Y$ hinge on the fact that we have enough co-projectives, i.e. each object in the category of left (resp. right) $C$-comodules admits a co-projective resolution. Since $C$ is counital one has $X \square_{C} C \cong X$ and $C \square_{C} Y \cong Y$ as $B$-modules meaning $C$ is co-projective in the category of $B$-equivariant $C$-comodules both left and right. Since $k$ is 
a field, $\mathrm{CB}_{*}^{\mathrm{bar}}(C) \underset{C}{\square} Y$ and $X \underset{C}{\square} \mathrm{CB}_{*}^{\mathrm{bar}}(C)$ will provide these resolutions for $Y$ and $X$ respectively. Then the derived functors of the cotorsion product exist and one can immediately see that $\operatorname{Cotor}_{*}^{C}(X, Y)$ are naturally $B$-modules after Lemma 2.4 and Proposition 2.3

Definition 2.7. The bar complex $\mathrm{CB}_{*}(C)$ associated with a coalgebra $C$ is defined as $\mathrm{CB}_{n}(C)=\mathrm{CB}_{n-1}^{\mathrm{bar}}(C)$ for any $n \geq 1$ and $\mathrm{CB}_{0}(C)=C$. The differentials too are the same for $n \geq 1$ and we let $d_{0}^{\mathrm{CB}}=\Delta_{C}$.

Definition 2.8. A coalgebra $C$ is called $\mathrm{H}$-counital if $\mathrm{CB}_{*}(C)$ is acyclic. This is equivalent to saying the bar resolution $\mathrm{CB}_{*}^{\text {bar }}(C)$ is quasi-isomorphic to the differential graded $k$-module $C$ concentrated at degree 0 .

\section{Twisted Cartier-Hochschild homology}

Let $C$ be a coalgebra. Define a coalgebra $C^{e}=C \otimes C^{o p}$ with the diagonal comultiplication

$$
\Delta\left(c \otimes c^{\prime}\right)=\left(c_{(1)} \otimes c_{(2)}^{\prime}\right) \otimes\left(c_{(2)} \otimes c_{(1)}^{\prime}\right)
$$

for any $c \otimes c^{\prime}$ from $C^{e}$.

It is easy to see that the categories of $C$-bicomodules and right $C^{e}$-comodules are isomorphic. Given any $C$-bicomodule $M$ the corresponding right $C^{e}$-comodule is again $M$ with comodule structure defined as

$$
\rho_{M}^{e}(m)=m_{(0)} \otimes\left(m_{(1)} \otimes m_{(-1)}\right)
$$

for any $m \in M$.

Assume $C$ is a $B$-module coalgebra and $X$ and $Y$ are left $B$-equivariant $C$-bicomodules. Note that unless $B$ is cocommutative, the cotensor product $X \underset{C^{e}}{\square} Y$ is not a $B$-module under the diagonal $B$ action.

Definition 3.1. Let $C$ be a coalgebra and $M$ be a $C$-bicomodule. Define a differential graded $k$-module $\mathrm{CH}_{*}(C, M)$ by letting $\mathrm{CH}_{n}(C, M)=M \otimes C^{\otimes n}$. We define a pre-simplicial structure

$$
\partial_{j}\left(m \otimes c^{1} \otimes \cdots \otimes c^{n}\right)= \begin{cases}m_{(0)} \otimes m_{(1)} \otimes c^{1} \otimes \cdots \otimes c^{n} & \text { if } j=0 \\ m \otimes c^{1} \otimes \cdots \otimes c_{(1)}^{j} \otimes c_{(2)}^{j} \otimes \cdots \otimes c^{n} & \text { if } 1 \leq j \leq n \\ m_{(0)} \otimes c^{1} \otimes \cdots \otimes c^{n} \otimes m_{(-1)} & \text { if } j=n+1\end{cases}
$$

and a differential $d_{n}^{\mathrm{CH}}=\sum_{j=0}^{n+1}(-1)^{j} \partial_{j}$.

Note that $\mathrm{CH}_{*}(C, M)$ is a differential graded $k$-module and a graded $B$-module but not a differential graded $B$-module unless $B$ is cocommutative. 
Theorem $3.2([8])$. Let $C$ be a counital coalgebra and $M$ be a $C$-bicomodule. Then there is an isomorphism of differential graded modules $\mathrm{CH}_{*}(C, M) \cong M \underset{C^{e}}{\square} \mathrm{CB}_{*}^{\text {bar }}(C)$

Corollary 3.3. Let $C$ be a counital coalgebra and $0 \rightarrow N \rightarrow M \rightarrow M / N \rightarrow 0$ be a short exact sequence of $C$-bicomodules. Then we have a short exact sequence of differential graded $k$-modules of the form

$$
0 \longrightarrow \mathrm{CH}_{*}(C, N) \rightarrow \mathrm{CH}_{*}(C, M) \rightarrow \mathrm{CH}_{*}(C, M / N) \rightarrow 0
$$

Proof. Since $\mathrm{CB}_{*}^{\mathrm{bar}}(C)$ consists of co-projective $C^{e}$-comodules we get a short exact sequence of the form

$$
0 \rightarrow N \underset{C^{e}}{\square} \mathrm{CB}_{*}^{\mathrm{bar}}(C) \rightarrow M \underset{C^{e}}{\square} \mathrm{CB}_{*}^{\mathrm{bar}}(C) \rightarrow M / N \underset{C^{e}}{\square} \mathrm{CB}_{*}^{\mathrm{bar}}(C) \rightarrow 0
$$

The result follows after Theorem 3.2

Remark 3.4. One can give a direct proof of Corollary 3.3 without appealing to ${\underset{C}{e}}_{\mathrm{C}} \mathrm{CB}_{*}^{\mathrm{bar}}(C)$. Indeed, for a direct proof one need not $C$ to be counital.

Definition 3.5. Let $X$ be a left $B$-comodule and $M$ be a $C$-bicomodule. Define $X \rtimes M$ as the $C^{e}$-comodule with $X \rtimes M=X \otimes M$ on the $k$-module level and we let

$$
\rho^{e}(x \otimes m)=\left(x_{(0)} \otimes m_{(0)}\right) \otimes\left(m_{(1)} \otimes x_{(-1)} m_{(-1)}\right)
$$

Note that

$$
\begin{aligned}
\left(\rho^{e} \otimes i d_{C^{e}}\right) \rho^{e}(x \otimes M) & =\left(x_{(0)(0)} \otimes m_{(0)(0)}\right) \otimes\left(m_{(0)(1)} \otimes x_{(0)(-1)} m_{(0)(-1)}\right) \otimes\left(m_{(1)} \otimes x_{(-1)} m_{(-11)}\right) \\
& =\left(x_{(0)} \otimes m_{(0)}\right) \otimes\left(m_{(1)} \otimes x_{(-1)} m_{(-1)}\right) \otimes\left(m_{(2)} \otimes x_{(-2)} m_{(-2)}\right) \\
& =\left(x_{(0)} \otimes m_{(0)}\right) \otimes\left(m_{(1)(1)} \otimes x_{(-1)(2)} m_{(-1)(2)}\right) \otimes\left(m_{(1)(2)} \otimes x_{(-1)(1)} m_{(-1)(1)}\right) \\
& =\left(i d_{X \rtimes C} \otimes \Delta\right) \rho^{e}(x \otimes m)
\end{aligned}
$$

for any $(x \otimes m)$ from $X \rtimes M$.

Definition 3.6. Assume $X$ is a $B$-module/comodule and $M$ is a $C$-bicomodule. We define the CartierHochschild complex of $C$ with coefficients in $M$ twisted by $X$ as

$$
\mathrm{CH}_{*}^{\mathrm{tw}}(C, M ; X):=\mathrm{CH}_{*}(C, X \rtimes M):=(X \rtimes M) \underset{C^{e}}{\square} \mathrm{CB}_{*}^{\mathrm{bar}}(C)
$$

In the special case where $M=C$ we will write $\mathrm{CH}_{*}^{\mathrm{tw}}(C ; X):=\mathrm{CH}_{*}^{\mathrm{tw}}(C, C ; X)$.

Proposition 3.7. Let $C$ be a counital B-module coalgebra and $M$ be a B-equivariant $C$-bicomodule. There is a graded $B$-module structure on $\mathrm{CH}_{*}^{\mathrm{tw}}(C, M ; X)$. 
Proof. We define a $B$-module structure on $\mathrm{CH}_{*}^{\mathrm{tw}}(C, M ; X)$ as follows: we let

$$
L_{b}\left(x \otimes m \otimes c^{1} \otimes \cdots \otimes c^{n}\right)=b_{(n+2)} x \otimes b_{(1)} m \otimes b_{(2)} c^{1} \otimes \cdots \otimes b_{(n+1)} c^{n}
$$

for any $n \geq 0, x \otimes m \otimes c^{1} \otimes \cdots \otimes c^{n}$ from $\mathrm{CH}_{n}^{\mathrm{tw}}(C, M ; X)$ and $b \in B$. One can easily check that the action is coassociative and $\left[L_{b}, \partial_{j}\right]=0$ for any $b \in B, 0 \leq n$ and $0 \leq j \leq n$. Note that, unless we have a prescribed interaction between the $B$-action and $B$-coaction on $X$, we can not infer anything about $\left[L_{b}, \partial_{n+1}\right]$.

Let us recall the following definition from [1].

Definition 3.8. Let $B$ be a Hopf algebra with an invertible antipode. Assume $X$ is a left $B$-module and a left $B$-comodule. $X$ is called an anti-Yetter-Drinfeld (aYD) module if one has

$$
(b x)_{(-1)} \otimes(b x)_{(0)}=b_{(1)} x_{(-1)} S^{-1}\left(b_{(3)}\right) \otimes b_{(2)} x_{(0)}
$$

for any $x \in X$ and $b \in B$.

Theorem 3.9. Let $B$ be a Hopf algebra with an invertible antipode and $X$ be an aYD module. Assume $C$ is a B-module coalgebra and $M$ is a B-equivariant $C$-bicomodule. Then $k \underset{B}{\otimes} \mathrm{CH}_{*}^{\mathrm{tw}}(C, M ; X)$ is a differential graded $k$-module with the induced differentials $i d \underset{B}{\otimes} d_{*}^{\mathrm{CH}}$.

Proof. Consider the graded $k$-submodule $I_{*}$ of $\mathrm{CH}_{*}^{\mathrm{tw}}(C, M ; X)$ generated by the commutators of the form $\left[L_{b}, d_{n}^{\mathrm{CH}}\right](\Psi)$ where $n \geq 0, \Psi \in \mathrm{CH}_{*}^{\mathrm{tw}}(C, M ; X)$ and $b \in B$. Let us show that $I_{*}$ is a differential graded $k$-submodule and also a graded $B$-submodule of $\mathrm{CH}_{*}^{\mathrm{tw}}(C, M ; X)$. Start with

$$
d_{n+1}^{\mathrm{CH}}\left[L_{b}, d_{n}^{\mathrm{CH}}\right]=-\left[L_{b}, d_{n+1}^{\mathrm{CH}}\right] d_{n}^{\mathrm{CH}}+\left[L_{b}, d_{n+1}^{\mathrm{CH}} d_{n}^{\mathrm{CH}}\right]=-\left[L_{b}, d_{n+1}^{\mathrm{CH}}\right] d_{n}^{\mathrm{CH}}
$$

which proves $I_{*}$ is a differential graded $k$-submodule. Then

$$
L_{y}\left[L_{b}, d_{n}^{\mathrm{CH}}\right]=-\left[L_{y}, d_{n}^{\mathrm{CH}}\right] L_{b}+\left[L_{b y}, d_{n}^{\mathrm{CH}}\right]
$$

which proves $I_{*}$ is a graded $B$-submodule. By design $\mathrm{CH}_{*}^{\mathrm{tw}}(C, M ; X) / I_{*}$ is a differential graded $B$-module. Therefore $k \underset{B}{\otimes}\left(\mathrm{CH}_{*}^{\mathrm{tw}}(C, M ; X) / I_{*}\right)$ is a differential graded $k$-module. The rest of the proof concentrates on proving $k \underset{B}{\otimes} I_{*}=0$ which would imply that the graded $k$-modules $k \underset{B}{\otimes}\left(\mathrm{CH}_{*}^{\mathrm{tw}}(C, M ; X) / I_{*}\right)$ and $k \underset{B}{\otimes}$ $\mathrm{CH}_{*}^{\mathrm{tw}}(C, M, X)$ are isomorphic. Note the remark we made in the proof of Proposition 3.7 and observe that $\left[L_{b}, d_{n}^{\mathrm{CH}}\right]=(-1)^{n+1}\left[L_{b}, \partial_{n+1}\right]$ for any $n \geq 0$. Take an arbitrary element $\Phi$ from $k \underset{B}{\otimes} I_{*}$ of the form 
$\sum_{i} 1 \underset{B}{\otimes}\left[L_{b^{i}}, \partial_{n+1}\right]\left(x^{i} \otimes m^{i} \otimes c^{1, i} \otimes \cdots \otimes c^{n, i}\right)$ and consider the equalities

$$
\begin{aligned}
& 1 \underset{B}{\otimes} \partial_{n+1} L_{b^{i}}\left(x^{i} \otimes m^{i} \otimes c^{1, i} \otimes \cdots \otimes c^{n, i}\right)=1 \underset{B}{\otimes} \partial_{n+1}\left(b_{(n+2)}^{i} x^{i} \otimes b_{(1)}^{i} m^{i} \otimes b_{(2)}^{i} c^{1, i} \otimes \cdots \otimes b_{(n+1)}^{i} c^{n, i}\right) \\
& =1 \underset{B}{\otimes} b_{(n+4)}^{i} x_{(0)}^{i} \otimes b_{(2)}^{i} m_{(0)}^{i} \otimes \otimes b_{(3)}^{i} c^{1, i} \otimes \cdots \otimes b_{(n+2)}^{i} c^{n, i} \otimes b_{(n+3)}^{i} x_{(-1)} S^{-1}\left(b_{(n+5)}^{i}\right) b_{(1)}^{i} m_{(-1)} \\
& =1 \underset{B}{\otimes} L_{b_{(2)}^{i}}\left(x_{(0)}^{i} \otimes m_{(0)}^{i} \otimes c^{1, i} \otimes \cdots \otimes c^{n, i} \otimes x_{(-1)} S^{-1}\left(b_{(3)}^{i}\right) b_{(1)}^{i} m_{(-1)}\right) \\
& =\varepsilon\left(b^{i}\right) \underset{B}{\otimes}\left(x_{(0)}^{i} \otimes m_{(0)}^{i} \otimes c^{1, i} \otimes \cdots \otimes c^{n, i} \otimes x_{(-1)} m_{(-1)}\right) \\
& =\varepsilon\left(b^{i}\right) \underset{B}{\otimes} \partial_{n+1}\left(x^{i} \otimes m^{i} \otimes c^{1, i} \otimes \cdots \otimes c^{n, i}\right)
\end{aligned}
$$

which means $\Phi=0$ as we wanted to show.

Theorem 3.10. Assume $B$ is a Hopf algebra with an invertible antipode and $X$ is an aYD module. Let $C$ be a counital $B$-module coalgebra and $0 \rightarrow N \rightarrow M \rightarrow M / N \rightarrow 0$ be a short exact sequence of $B$ equivariant $C$-bicomodules. Assume $N \rightarrow M$ is a split injective morphism of B-modules. Then there is a short exact sequence of differential graded $k$-modules of the form

$$
0 \rightarrow k \underset{B}{\otimes} \mathrm{CH}_{*}^{\mathrm{tw}}(C, N ; X) \rightarrow k \underset{B}{\otimes} \mathrm{CH}_{*}^{\mathrm{tw}}(C, M ; X) \longrightarrow k \underset{B}{\otimes} \mathrm{CH}_{*}^{\mathrm{tw}}(C, M / N ; X) \rightarrow 0
$$

Proof. Corollary 3.3 implies we have a short exact sequence of differential graded $k$-modules and graded $B$-modules

$$
0 \rightarrow \mathrm{CH}_{*}^{\mathrm{tw}}(C, N ; X) \rightarrow \mathrm{CH}_{*}^{\mathrm{tw}}(C, M ; X) \rightarrow \mathrm{CH}_{*}^{\mathrm{tw}}(C, M / N ; X) \rightarrow 0
$$

Upon tensoring with $k$ over $B$ we get the short exact sequence in Equation 3.1 as a short exact sequence of graded $k$-modules. Theorem 3.9 tells us that each of the terms in the short exact sequence is a differential graded $k$-module. Since the differentials are induced the short exact sequence in Equation 3.1 is a short exact sequence of differential graded $k$-modules.

\section{EXCISION IN HOPF CYCLIC HOMOLOGY}

In this section we assume $B$ is a Hopf algebra with an invertible antipode and $X$ is an aYD module unless otherwise stated.

Lemma 4.1. Let $P_{1}, \ldots, P_{n}$ be a finite collection of projective $B$-modules. Then $P_{1} \otimes \cdots \otimes P_{n}$ is projective with diagonal B-action.

Proof. Since every projective module is a direct summand of a free module, and direct sum of projective modules is projective, the statement reduces to proving $B^{\otimes n}$ is projective. Consider the endomorphism of 
$k$-modules $B^{\otimes n} \stackrel{\Gamma}{\longrightarrow} B^{\otimes n}$ defined by

$$
\Gamma_{n}\left(b^{1} \otimes \cdots \otimes b^{n}\right)=b_{(1)}^{1} \otimes b_{(2)}^{1} b_{(1)}^{2} \otimes \cdots \otimes b_{(n)}^{1} b_{(n-1)}^{2} \cdots b_{(2)}^{n-1} b^{n}
$$

Note that $\Gamma_{n}\left(b b^{1} \otimes \cdots \otimes b^{n}\right)=L_{b} \Gamma_{n}\left(b^{1} \otimes \cdots \otimes b^{n}\right)$ for any $b \in B$ and $\left(b^{1} \otimes \cdots \otimes b^{n}\right)$ from $B^{\otimes n}$. $\Gamma$ has an inverse given by

$$
\Gamma_{n}^{-1}\left(b^{1} \otimes \cdots \otimes b^{n}\right)=b_{(1)}^{1} \otimes S\left(b_{(2)}^{1}\right) b_{(1)}^{2} \otimes \cdots \otimes S\left(b_{(2)}^{n-1}\right) b^{n}
$$

which means $B^{\otimes n}$ with diagonal $B$-action is isomorphic to the $B$-module $B^{\otimes n}$ with $B$ acting on the first tensor component. Since $k$ is a field, the second $B$-module is $B$-free. The result follows.

Lemma 4.2. Given a left $B-$ module $U$ define right $B-$ module $U^{o p}$ by letting $u \cdot b:=S^{-1}(b) u$ for any $u \in U$ and $b \in B$. Then given any other left $B$-module $V$ there is an isomorphism of $k$-modules

$$
k \underset{B}{\otimes}(U \otimes V) \cong U^{o p}{\underset{B}{\otimes} V} V
$$

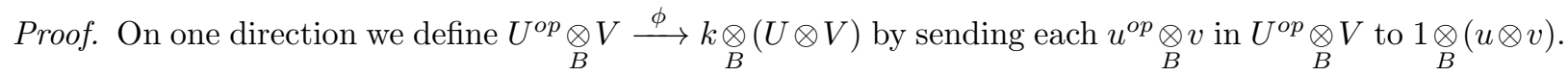
We must show that $\phi$ is well-defined. For that, for any $\left(u^{o p} b \underset{B}{\otimes} v\right)$ from $U^{o p} \underset{B}{\otimes} V$ we consider

$$
\phi\left(u^{o p} b \underset{B}{\otimes} v\right)=1 \underset{B}{\otimes}\left(S^{-1}(b) u \otimes v\right)=1 \underset{B}{\otimes} S^{-1}\left(b_{(2)}\right)\left(u \otimes b_{(1)} v\right)=1{\underset{B}{\otimes}}_{B}(u \otimes b v)=\phi\left(u^{o p} \otimes_{B} b v\right)
$$

which proves $\phi$ is well-defined. Conversely take $1 \underset{B}{\otimes}(u \otimes v)$ from $k \underset{B}{\otimes}(U \otimes V)$ and let $\psi(1 \underset{B}{\otimes}(u \otimes v))$ be $\left(u^{o p} \otimes_{B} v\right)$. In order $\psi$ be well-defined we must have

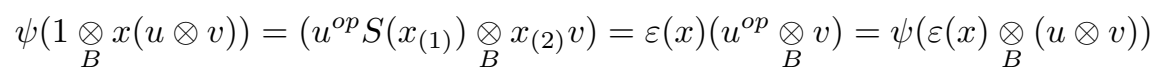

we we wanted to show. One can easily see that $\psi$ and $\phi$ are mutual inverses.

Lemma 4.3. Let $\mathcal{A}_{*}$ be an acyclic differential graded $B$-module where each $\mathcal{A}_{n}$ is $B$-projective. Let $U$ be a $B$-module with finite projective dimension and let $V$ be a projective $B-$ module. Then $k \otimes\left(U \otimes \mathcal{A}_{*} \otimes V\right)$ is acyclic.

Proof. We will show that $U_{B}^{o p} \underset{B}{\otimes}\left(\mathcal{A}_{*} \otimes V\right)$ is acyclic which after using Lemma 4.2 would imply $k \underset{B}{\otimes}\left(U \otimes \mathcal{A}_{*} \otimes V\right)$ is acyclic. Note that $\mathcal{A}_{*} \otimes V$ is an acyclic differential graded $B$-module which consists of projective $B$ modules following Lemma 4.1 Since each $\mathcal{A}_{n} \otimes V$ is projective, the differential graded $B$-module $\mathcal{A}_{n} \otimes V$ is its own Cartan-Eilenberg resolution. Then the hyper Tor products are given by

$$
\operatorname{Tor}_{*}^{B}\left(U^{o p}, \mathcal{A}_{*} \otimes V\right)=H_{*}\left(U^{o p} \underset{B}{\otimes}\left(\mathcal{A}_{*} \otimes V\right)\right)
$$


Since we actually compute the hyper Tor functors, one can compute the homology of $U^{o p} \underset{B}{\otimes}\left(\mathcal{A}_{*} \otimes V\right)$ by taking a $B$-projective resolution $\left(\mathcal{U}_{*}, d_{*}^{\mathcal{U}}\right)$ of $U^{o p}$ and then compute the homology of the double complex $\mathcal{U}_{*} \underset{B}{\otimes}\left(\mathcal{A}_{*} \otimes V\right)$. Since we did not assume $\mathcal{A}_{*}$ is bounded, there is a problem about the convergence of the canonical spectral sequence

$$
E_{p q}^{2}=H_{p}\left(\mathcal{U}_{*} \underset{B}{\otimes}\left(H_{q}\left(\mathcal{A}_{*}, d_{*}^{\mathcal{A}}\right) \otimes V\right), d_{*}^{\mathcal{U}}\right)
$$

coming from this double complex. However, since we assumed $U$ is of finite projective dimension, the double complex $\mathcal{U}_{*} \underset{B}{\otimes}\left(\mathcal{A}_{*} \otimes V\right)$ is concentrated on an infinite horizontal strip which forces the spectral sequence to converge. Since $\mathcal{A}_{*}$ is acyclic, the spectral sequence is all 0. Thus $U^{o p} \underset{B}{\otimes}\left(\mathcal{A}_{*} \otimes V\right)$ is acyclic.

Theorem 4.4. Let $0 \rightarrow K \rightarrow C \rightarrow C / K \rightarrow 0$ be a short exact sequence of $B$-module coalgebras. Assume that both $C$ and $C / K$ are $B$-projective and $X$ has finite projective dimension. Assume also that $C / K$ is $H$-counital. Then the canonical epimorphism $C \rightarrow C / K$ of $B$-module coalgebras induces a weak equivalence of differential graded $k$-modules of the form $k \underset{B}{\otimes} \mathrm{CH}_{*}^{\mathrm{tw}}(C, C / K ; X) \rightarrow k \underset{B}{\otimes} \mathrm{CH}_{*}^{\mathrm{tw}}(C / K ; X)$.

Proof. First note that since $C / K$ is projective the short exact sequence of $B$-module coalgebras $0 \rightarrow K \rightarrow$ $C \rightarrow C / K \rightarrow 0$ is a split short exact sequence of $B$-modules. Define a sequence $\left\{G_{*}^{p}\right\}_{p \geq 0}$ of differential graded $k$-modules by letting

$$
G_{*}^{p}= \begin{cases}X \otimes(C / K) \otimes C^{\otimes n} & \text { if } n \leq p \\ X \otimes(C / K)^{\otimes n-p+1} \otimes C^{\otimes p} & \text { if } n>p\end{cases}
$$

The pre-simplicial structure for $n<p$ comes from $\mathrm{CH}_{*}^{\mathrm{tw}}(C, C / K ; X)$. For $n \geq p$

$$
\partial_{j}= \begin{cases}\left(i d_{X} \otimes i d_{j} \otimes \Delta_{C / K} \otimes i d_{n-j}\right) & \text { if } 0 \leq j \leq n-p \\ \left(i d_{X} \otimes i d_{n-p+1} \otimes \pi_{C / K} \otimes i d_{p}\right)\left(i d_{X} \otimes i d_{j} \otimes \Delta_{C} \otimes i d_{n-j}\right) & \text { if } n-p+1 \leq j \leq n \\ \left(i d_{X} \otimes i d_{n-p+1} \otimes \pi_{C / K} \otimes i d_{p}\right)\left(i d_{X} \otimes \tau_{n+1}\right)\left(\rho_{C / K} \otimes i d_{n}\right) & \text { if } j=n+1\end{cases}
$$

where $C \stackrel{\pi_{C / K}}{\longrightarrow} C / K$ is the canonical epimorphism and $\tau_{n+1}$ is the twisted cyclic permutation of length $n+2$ and $C / K \stackrel{\rho_{C / K}}{\longrightarrow} C \otimes C / K$ is the left $C$-comodule structure on $C / K$. There are epimorphisms of differential graded $k$-modules $G_{*}^{p+1} \stackrel{\pi_{*}^{p}}{\longrightarrow} G_{*}^{p}$ defined for any $p \geq 0$ where

$$
\pi_{n}^{p}= \begin{cases}\left(i d_{X} \otimes i d_{n+1}\right) & \text { if } n \leq p \\ \left(i d_{X} \otimes i d_{n-p} \otimes \pi_{C / K} \otimes i d_{p}\right) & \text { if } n>p\end{cases}
$$


with kernel

$$
\operatorname{ker}\left(\pi_{*}^{p}\right)= \begin{cases}0 & \text { if } n \leq p \\ X \otimes(C / K)^{\otimes n-p} \otimes K \otimes C^{\otimes p} & \text { if } n>p\end{cases}
$$

which is isomorphic as graded $B$-modules to $X \otimes \mathrm{CB}_{*}(C / K)[-p] \otimes K \otimes C^{\otimes p}$. Now, applying the functor $k \underset{B}{\otimes}$. we get a short exact sequence

$$
0 \rightarrow k \underset{B}{\otimes} \operatorname{ker}\left(\pi_{*}^{p}\right) \rightarrow k \underset{B}{\otimes} G_{*}^{p+1} \stackrel{i d \otimes \pi_{*}^{p}}{\longrightarrow} k \underset{B}{\otimes} G_{*}^{p} \rightarrow 0
$$

of graded $k$-modules by using the fact that the inclusion $K \rightarrow C$ is $B$-split. Theorem 3.9 implies $k \underset{B}{\otimes} G_{*}^{p}$ is a differential graded $k$-module with the induced differentials. Since $C / K$ is $\mathrm{H}$-counital Lemma 4.1 and Lemma 4.3 implies $k \underset{B}{\otimes} k \operatorname{ker}\left(\pi_{*}^{p}\right)$ is acyclic. Now observe that $k \underset{B}{\otimes} G_{*}^{0}=k \underset{B}{\otimes} \mathrm{CH}^{\mathrm{tw}}(C / K ; X)$ and $k \underset{B}{\otimes} G_{n}^{p}=k \underset{B}{\otimes} \mathrm{CH}_{n}^{\mathrm{tw}}(C, C / K ; X)$ for $n>p$. The result follows.

Proposition 4.5. Let $0 \rightarrow K \rightarrow C \rightarrow C / K \rightarrow 0$ be a short exact sequence of $B$-module coalgebras. Assume that $C / K$ is $H$-counital. We define a differential graded $B$-module $\mathrm{C}_{*}(K, C / K)$ by letting $\mathrm{C}_{n}(K, C / K)=K \otimes C^{\otimes n} \otimes C / K$ then defining the pre-simplicial structure as

$$
\partial_{j}= \begin{cases}\Delta_{K} \otimes i d_{n+1} & \text { if } j=0 \\ i d_{j+1} \otimes \Delta_{C} \otimes i d_{n-j+1} & \text { if } 0<j<n+1 \\ i d_{n+1} \otimes \rho_{C / K} & \text { if } j=n+1\end{cases}
$$

where $\Delta_{C}$ and $\Delta_{K}$ are the comultiplication on $C$ and $K$ respectively, and $C / K \stackrel{\rho_{C / K}}{\longrightarrow} C \otimes C / K$ is the $C$-comodule structure on $C / K$. Then $\mathrm{C}_{*}(K, C / K)$ is an acyclic differential graded $B$-module.

Proof. The fact that $\mathrm{C}_{*}(K, C / K)$ is a differential graded $B$-module is easy to see. Consider the sequence of differential graded $B$-modules

$$
J_{*}^{p}= \begin{cases}K \otimes C^{\otimes n} \otimes C / K & \text { if } n \leq p \\ K \otimes C^{\otimes p} \otimes(C / K)^{\otimes n-p+1} & \text { if } n>p\end{cases}
$$

The differentials on $J_{*}^{p}$ descend from $\mathrm{C}_{*}(K, C / K)$ for $n<p$. For $n \geq p$ we define a pre-simplicial structure

$$
\partial_{j}= \begin{cases}\left(i d_{p+1} \otimes \pi_{C / K} \otimes i d_{n-p}\right)\left(\Delta_{K} \otimes i d_{n+1}\right) & \text { if } j=0 \\ \left(i d_{p+1} \otimes \pi_{C / K} \otimes i d_{n-p}\right)\left(i d_{j} \otimes \Delta_{C} \otimes i d_{n-j+1}\right) & \text { if } 1 \leq j \leq p \\ \left(i d_{j} \otimes \Delta_{C / K} \otimes i d_{n-j+1}\right) & \text { if } p<j \leq n+1\end{cases}
$$


There are epimorphisms of differential graded $B$-modules $J_{*}^{p+1} \stackrel{\pi_{*}^{p}}{\longrightarrow} J_{*}^{p}$ defined by

$$
\pi_{n}^{p}= \begin{cases}i d_{n+2} & \text { if } n \leq p \\ i d_{p+1} \otimes \pi_{C / K} \otimes i d_{n-p} & \text { if } n>p\end{cases}
$$

with kernels

$$
\operatorname{ker}\left(\pi_{*}^{p}\right)= \begin{cases}0 & \text { if } n \leq p \\ K \otimes C^{\otimes p} \otimes K \otimes(C / K)^{\otimes n-p} & \text { if } n>p\end{cases}
$$

which is isomorphic to the differential graded $B$-module $K \otimes C^{\otimes p} \otimes K \otimes \mathrm{CB}_{*}(C / K)[-p]$. Since $C / K$ is $\mathrm{H}$-counital, we see that each $\operatorname{ker}\left(\pi_{*}^{p}\right)$ is acyclic implying $\pi_{*}^{p}$ is a weak equivalence. For $p=0$ we have $J_{*}^{0} \cong K \otimes \mathrm{CB}_{*}(C / K)$ and for $p>n$ we have $J_{n}^{p}=\mathrm{C}_{n}(K, C / K)$. The result follows from the fact that $C / K$ is $\mathrm{H}-$ counital.

Theorem 4.6. Let $0 \rightarrow K \longrightarrow C \longrightarrow C / K \longrightarrow 0$ be a short exact sequence of $H$-counital $B$-module coalgebras. Assume that $X$ has finite projective dimension and both $C$ and $C / K$ are projective $B$-modules. Then the canonical inclusion of differential graded $k$-modules $k \underset{B}{\otimes} \mathrm{CH}_{*}^{\mathrm{tw}}(K ; X) \longrightarrow k \underset{B}{\otimes} \mathrm{CH}_{*}^{\mathrm{tw}}(C, K ; X)$ is a weak equivalence.

Proof. Define a sequence $\left\{F_{*}^{p}\right\}_{p \geq 0}$ of differential graded $k$-submodules of $\mathrm{CH}_{*}^{\mathrm{tw}}(C, K ; X)$ by letting

$$
F_{n}^{p}= \begin{cases}X \otimes K^{\otimes n+1} & \text { if } n \leq p \\ X \otimes K \otimes C^{\otimes n-p} \otimes K^{\otimes p} & \text { if } n>p\end{cases}
$$

There are inclusions $F_{*}^{p+1} \subseteq F_{*}^{p}$ and we see that

$$
F_{n}^{p} / F_{n}^{p+1}= \begin{cases}0 & \text { if } n \leq p \\ X \otimes K \otimes C^{\otimes n-p-1} \otimes C / K \otimes K^{\otimes p} & \text { if } n>p\end{cases}
$$

which is isomorphic to the differential graded $B$-module $X \otimes \mathrm{C}_{*}(K, C / K)[-p-1] \otimes K^{\otimes p}$. Since $K \rightarrow C$ is $B$-split we have a short exact sequence of differential graded $k$-modules of the form

$$
0 \rightarrow k \underset{B}{\otimes} F_{*}^{p+1} \rightarrow k \underset{B}{\otimes} F_{*}^{p} \rightarrow k \underset{B}{\otimes}\left(F_{*}^{p} / F_{*}^{p+1}\right) \rightarrow 0
$$

Now use Lemma 4.3 to conclude that $k \underset{B}{\otimes}\left(F_{*}^{p} / F_{*}^{p+1}\right)$ is acyclic. The result follows after observing $F_{*}^{0}=$ $\mathrm{CH}_{*}^{\mathrm{tw}}(C, K ; X)$ and for $n>p$ and $F_{n}^{p}=\mathrm{CH}_{n}^{\mathrm{tw}}(K ; X)$,

Definition 4.7. Assume $X$ is a left $B$-module $B \otimes X \stackrel{\mu_{X}}{\longrightarrow} X$ and a left $B$-comodule $X \stackrel{\rho_{X}}{\longrightarrow} B \otimes X$. We will call $X$ stable if $\mu_{X} \rho_{X}=i d_{X}$. In other words $x_{(-1)} x_{(0)}=x$ for any $x \in X$. 
Example 4.8. (1) Assume $X$ is a $B$-comodule. One can force $X$ to be a left $B$-module via the counit $B \stackrel{\varepsilon}{\longrightarrow} k$, i.e. $b(x)=\varepsilon(b) x$ for any $x \in X$ and $b \in B$. Then $X$ is a stable $B$-module/comodule. We will denote this stable module/comodule by $X_{\varepsilon}$.

(2) Similarly, if $X$ is a $B$-module, one can force $X$ to be a left $B$-comodule via $\rho(x)=(\mathbb{I} \otimes x)$. Then $X$ is a stable $B$-module/comodule. We will denote this stable module/comodule by $X_{\mathbb{I}}$.

(3) Assume $B$ is a Hopf algebra with an invertible antipode. We will consider $B$ as a left $B$-module via the left regular representation $b(x)=b x$ and a left $B$-comodule via the left co-adjoint coaction $x_{(-1)} \otimes x_{(0)}=x_{(1)} S^{-1}\left(x_{(3)}\right) \otimes x_{(2)}$. We will denote this $B$-module/comodule by $B^{\mathrm{r}, \text { ad }}$. Then

$$
x_{(-1)} x_{(0)}=x_{(1)} S^{-1}\left(x_{(3)}\right) x_{(2)}=x
$$

for any $x \in B$, i.e. $B^{r, a d}$ is a stable $B$-module comodule. Moreover

$$
\begin{aligned}
(y x)_{(-1)} \otimes(y x)_{(0)} & =y_{(1)} x_{(1)} S^{-1}\left(x_{(3)}\right) S^{-1}\left(y_{(3)}\right) \otimes y_{(2)} x_{(2)} \\
& =y_{(1)} x_{(-1)} S^{-1}\left(y_{(3)}\right) \otimes y_{(2)} x_{(0)}
\end{aligned}
$$

for any $x, y \in B$ meaning $B^{\mathrm{r} \text {,ad }}$ is a stable anti-Yetter-Drinfeld (SaYD) module.

(4) Assume $B$ is a Hopf algebra with an invertible antipode. Consider $B$ as a $B$-module via the left adjoint action $b(x)=b_{(1)} x S^{-1}\left(b_{(2)}\right)$ and as a $B$-comodule via the left regular co-representation $\left.x_{(-1)} \otimes x_{(0)}\right):=x_{(1)} \otimes x_{(2)}$. We will denote this $B$-module comodule by $B^{\text {ad, } \mathrm{r}}$. Then

$$
x_{(-1)} x_{(0)}=x_{(1)} x_{(3)} S^{-1}\left(x_{(2)}\right)=x
$$

for any $x \in B$. This means $B^{a d, r}$ is a stable $B$-module/comodule. Moreover, $B^{a d, r}$ is also a SaYD module since

$$
\begin{aligned}
(y x)_{(-1)} \otimes(y x)_{(0)} & =\left(y_{(1)} x S^{-1}\left(y_{(2)}\right)\right)_{(1)} \otimes\left(y_{(1)} x S^{-1}\left(y_{(2)}\right)\right)_{(2)} \\
& =y_{(1)} x_{(1)} S^{-1}\left(y_{(4)}\right) \otimes y_{(2)} x_{(2)} S^{-1}\left(y_{(3)}\right) \\
& =y_{(1)} x_{(-1)} S^{-1}\left(y_{(3)}\right) \otimes y_{(2)} x_{(0)}
\end{aligned}
$$

for any $x, y \in B$.

Remark 4.9. In [12] we introduced a para-cocyclic $B$-module $\mathbb{P C M}_{*}^{c}(C, B, X)$ and a cocyclic module $\mathbb{C M}_{*}^{c}(C, X):=k \underset{B}{\otimes} \mathbb{P C M}_{*}^{c}(C, B, X)$ of a triple $(C, B, X)$ where $B$ is a bialgebra, $C$ is a $B$-module algebra and $X$ is a stable $B$-module/comodule. We also showed that in case $B$ is a Hopf algebra with an invertible antipode and $X$ is a stable aYD module then one has

$$
\mathcal{C}_{*}^{B}(C ; X) \cong \mathbb{C M}_{*}^{c}(C, B, X):=k \underset{B}{\otimes} \mathbb{P C M}_{*}^{c}(C, B, X)
$$


where $\mathcal{C}_{*}^{B}(C ; X)$ is the cocyclic complex of [10] associated with the coalgebra $B$-module coalgebra $C$. $\mathcal{C}_{*}^{B}(C ; X)$ is a cocyclic complex whose Hochschild complex is $k \underset{B}{\otimes} \mathrm{CH}_{*}^{\mathrm{tw}}(C ; X)$ and whose bar complex is $k \underset{B}{\otimes}\left(X \otimes \mathrm{CB}_{*}(C)\right)$ with induced differentials.

Definition 4.10. A sequence of differential graded (resp. simplicial, cosimplicial, cyclic, cocyclic) modules $\mathcal{X}_{*} \stackrel{u_{*}}{\longrightarrow} \mathcal{Y}_{*} \stackrel{v_{*}}{\longrightarrow} \mathcal{Z}_{*}$ is called a homotopy cofibration sequence if there are connecting morphisms $H_{n+1}\left(\mathcal{Z}_{*}\right) \stackrel{\delta_{n}}{\longrightarrow} H_{n}\left(\mathcal{X}_{*}\right)$ (resp. $H_{n}\left(\mathcal{Z}_{*}\right) \stackrel{\delta_{n}}{\longrightarrow} H_{n+1}\left(\mathcal{X}_{*}\right)$ for cosimplicial and cocyclic modules) which fit into a long exact sequence of the form

$$
\ldots \stackrel{H_{n+1}\left(v_{*}\right)}{\longrightarrow} H_{n+1}\left(\mathcal{Z}_{*}\right) \stackrel{\delta_{n}}{\longrightarrow} H_{n}\left(\mathcal{X}_{*}\right) \stackrel{H_{n}\left(u_{*}\right)}{\longrightarrow} H_{n}\left(\mathcal{Y}_{*}\right) \stackrel{H_{n}\left(v_{*}\right)}{\longrightarrow} H_{n}\left(\mathcal{Z}_{*}\right) \stackrel{\delta_{n}}{\longrightarrow} \cdots
$$

or respectively for cosimplicial and cocyclic modules of the form

$$
\ldots \stackrel{H_{n-1}\left(v_{*}\right)}{\longrightarrow} H_{n-1}\left(\mathcal{Z}_{*}\right) \stackrel{\delta_{n-1}}{\longrightarrow} H_{n}\left(\mathcal{X}_{*}\right) \stackrel{H_{n}\left(u_{*}\right)}{\longrightarrow} H_{n}\left(\mathcal{Y}_{*}\right) \stackrel{H_{n}\left(v_{*}\right)}{\longrightarrow} H_{n}\left(\mathcal{Z}_{*}\right) \stackrel{\delta_{n}}{\longrightarrow} \cdots
$$

for any $n \geq 0$.

Theorem 4.11 (Excision in Hopf Cyclic Homology). Let B be a Hopf algebra with an invertible antipode and $X$ be a stable anti-Yetter-Drinfeld (SaYD) module. Let $C$ be a counital $B$-module coalgebra and $0 \longrightarrow$ $K \rightarrow C \rightarrow C / K \rightarrow 0$ be a short exact sequence of $H$-counital $B$-module coalgebras. Assume that both $C$ and $C / K$ are $B$-projective and $X$ has finite projective dimension. Then there is a homotopy cofibration sequence of the form

$$
\mathbb{C M}_{*}^{c}(K, B, X) \rightarrow \mathbb{C M}_{*}^{c}(C, B, X) \rightarrow \mathbb{C M}_{*}^{c}(C / K, B, X)
$$

Proof. Theorem 3.10 Theorem 4.4 and Theorem 4.6 tell us we have a homotopy cofibration sequence of the form

$$
k \underset{B}{\otimes} \mathrm{CH}_{*}^{\mathrm{tw}}(K ; X) \rightarrow k \underset{B}{\otimes} \mathrm{CH}_{*}^{\mathrm{tw}}(C ; X) \rightarrow k \underset{B}{\otimes} \mathrm{CH}_{*}^{\mathrm{tw}}(C / K ; X)
$$

This is equivalent to saying Hopf Hochschild homology has excision. Since $K, C$ and $C / K$ are all $\mathrm{H}_{-}$ counital and $B$-projective, the differential graded $k$-complexes $k \underset{B}{\otimes}\left(X \otimes \mathrm{CB}_{*}(K)\right), k \underset{B}{\otimes}\left(X \otimes \mathrm{CB}_{*}(C)\right)$ and $k \underset{B}{\otimes}\left(X \otimes \mathrm{CB}_{*}(C / K)\right)$ are all acyclic because $X$ has finite projective dimension following Lemma 4.3 Therefore we have another homotopy cofibration sequence

$$
k \underset{B}{\otimes}\left(X \otimes \mathrm{CB}_{*}(K)\right) \rightarrow k \underset{B}{\otimes}\left(X \otimes \mathrm{CB}_{*}(C)\right) \rightarrow k \underset{B}{\otimes}\left(X \otimes \mathrm{CB}_{*}(C / K)\right)
$$

Since $\mathbb{C M}_{*}^{c}(\cdot ; X)$ is composed of $k \underset{B}{\otimes} \mathrm{CH}_{*}^{\mathrm{tw}}(\cdot ; X)$ and $k \underset{B}{\otimes}\left(X \otimes \mathrm{CB}_{*}(\cdot)\right)$ with induced cocyclic structures the result follows. 
Corollary 4.12. Assume $B$ is a Hopf algebra with an invertible antipode and $X$ be a SaYD module. Let $\left\{C_{i}\right\}_{i \in \Lambda}$ be a class of counital B-module coalgebras such that each $C_{i}$ is $B$-projective. Let $C=\bigoplus_{i \in \Lambda} C_{i}$ be the direct sum considered as a counital B-module coalgebra via the direct sum of comultiplication structures. Then

$$
H C_{*}(C ; X) \cong \bigoplus_{i \in \Lambda} H C_{*}\left(C_{i} ; X\right)
$$

In other words cyclic homology is additive on the class of B-projective counital B-module coalgebras.

Remark 4.13. If we drop $B$, in other words if we set $B=k$, then Theorem 4.11 reduces to a Wodzicki type excision theorem [9] for coalgebras. We suspect that we have a Cuntz-Quillen type of excision [7] in periodic equivariant cyclic homology of coalgebras, if we drop the H-counitality condition.

Conjecture 4.14. Let $B$ be a Hopf algebra with an invertible antipode and $X$ be a SaYD module. Let $0 \rightarrow K \longrightarrow C \longrightarrow C / K \longrightarrow 0$ be a sequence of $H$-counital $B$-module coalgebras such that both $C$ and $C / K$ are B-projective. Then one has a 6-term exact sequence in periodic cyclic homology of the form

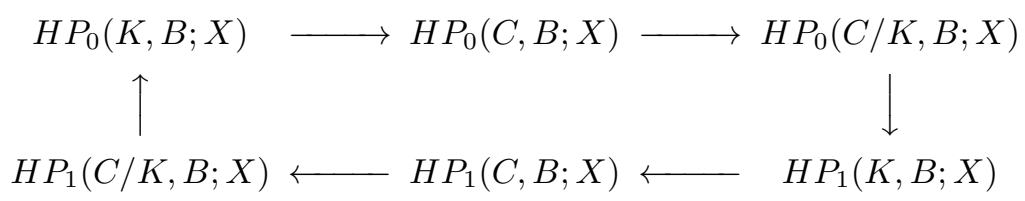

\section{Co-Integrals And Finite PRoJeCtive Dimension}

The following proposition gives us a good example of a Hopf algebra and a coefficient module with finite projective dimension.

Proposition 5.1. If $B$ is $U(\mathfrak{g})$ the universal enveloping algebra of a finite dimensional Lie algebra, then the trivial $\mathfrak{g}$-module $k$ has finite projective dimension.

Proof. The Chevalley-Eilenberg complex $\mathrm{CE}_{*}(\mathfrak{g})=U(\mathfrak{g}) \otimes \Lambda^{*} \mathfrak{g}$ of $\mathfrak{g}$ with coefficients in $U(\mathfrak{g})$ is a $U(\mathfrak{g})$ projective resolution of $k$. Since $\mathfrak{g}$ is finite dimensional, $\mathrm{CE}_{*}(\mathfrak{g})$ is a finite resolution.

In the rest of this section, we investigate the conditions which would make $k_{(1, \epsilon)}$ into a projective $B^{-}$ module.

Recall that an element $\sigma \in B$ is called a normalized right co-integral if one has $\sigma b=\varepsilon(b) \sigma$ and $\varepsilon(\sigma)=1$ for any $b \in B$. It is well-known [16] that if $B$ is a Hopf algebra then $B$ admits a right (or equivalently a left) co-integral iff $B$ is semi-simple. 
The following fact is easy to prove, yet its proof is given to demonstrate the connection between the $B$-projectivity of $k$ and existence of a co-integral.

Proposition 5.2. $k$ is a projective right $B$-module iff $B$ admits a normalized right co-integral.

Proof. Assume $k$ is projective right $B$-module. Then since any epimorphism of right $B$-modules of the form $Z \stackrel{p}{\longrightarrow} k$ splits, so does $B \stackrel{\varepsilon}{\longrightarrow} k$. Let us denote the splitting by $k \stackrel{s}{\longrightarrow} B$. Therefore there is an element $\sigma=s(1)$ such that $\sigma b=s(1) b=s(\varepsilon(b) 1)=e=\varepsilon(b) \sigma$ for any $b \in B$ implying $\sigma$ is a right co-integral Conversely, assume $B$ admits a right co-integral $\sigma \in B$ and consider an arbitrary epimorphism of right $B$-modules of the form $Z \stackrel{p}{\longrightarrow} k$. Pick an element $z \in Z$ such that $p\left(z_{1}\right)=1$. Since $p$ is non-trivial and $k$ is a field, such an element exists. Define $k \stackrel{s}{\longrightarrow} Z$ by letting $s(\lambda)=\lambda z_{1} \sigma$ for any $\lambda \in k$. Then $s$ is a morphism of $B$-modules since

$$
s(\lambda b)=\lambda \varepsilon(b) s(1)=\lambda \varepsilon(b) z_{1} \sigma=\lambda z_{1} \sigma b=s(\lambda) b
$$

Moreover,

$$
p(s(\lambda))=\lambda p\left(z_{1} \sigma\right)=\lambda p\left(z_{1}\right) \varepsilon(\sigma)=\lambda
$$

implying $s$ is a right $B$-module splitting of $p$. Result follows.

Corollary 5.3. If $B$ is a Hopf algebra admitting a normalized right or left co-integral and $C$ is any counital $B$-module coalgebra, then for any $S a Y D$ module $X$ and for any short exact sequence of $H$-counital $B$-module coalgebras $0 \rightarrow K \rightarrow C \longrightarrow C / K \rightarrow 0$ one has a homotopy cofibration sequence given in Equation 4.1

\section{Relative BialgeBRa CYClic homology}

Definition 6.1. Given a $B$-module coalgebra $C$ and a $B$-submodule subcoalgebra $K$, we define the relative cyclic homology of the pair $(C, K)$ with coefficients in a stable $B$-module/comodule $X$ as the cyclic homology of the cyclic $k$-module $\mathbb{C M}_{*}^{c}((C, K), B, X)$ which is defined as

$$
\operatorname{coker}\left(\mathbb{C M}_{*}^{c}(K, B, X) \stackrel{\mathbb{C M}_{*}^{c}(i)}{\longrightarrow} \mathbb{C M}_{*}^{c}(C, B, X)\right)
$$

the cokernel of the canonical morphism of cocyclic $k$-modules $\mathbb{C M}_{*}^{c}(K, B, X) \stackrel{\mathbb{C M}_{*}^{c}(i)}{\longrightarrow} \mathbb{C M}_{*}^{c}(C, B, X)$ coming from the inclusion $K \rightarrow C$.

Remark 6.2. The canonical morphism $\mathbb{C M}_{*}^{c}(K, B, X) \rightarrow \mathbb{C M}_{*}^{c}(C, B, X)$ is not necessarily a monomorphism of cocyclic $k$-modules. When $K \rightarrow C$ is $B$-split as a morphism of $B$-modules this canonical inclusion is a monomorphism of cocyclic $k$-modules. 
Definition 6.3. Given a $B$-module coalgebra $C$ and a $B$-submodule coideal $J$, we define the relative cyclic homology of the pair $(C, J)$ with coefficients in a stable $B$-module/comodule $X$ as the cyclic homology of the triple $(C / J, B, X)$.

Remark 6.4. Assume $C$ is a $B$-module coalgebra. Any $B$-submodule subcoalgebra $K$ is also a $B$ submodule coideal which means there are potentially two different versions of relative cyclic homology of the pair $(C, K)$.

Remark 6.5. In [6] Connes and Moscovici defined the relative Hopf cyclic homology of a Hopf algebra $H$ and a Hopf subalgebra $K$ with coefficients in a SaYD module $X$ as the Hopf cyclic homology of the triple $(H \underset{K}{\otimes} k, H, X)$. This is the same as the relative cyclic homology of the pair $\left(H, H K^{+}\right)$with coefficients in $X$ where $H K^{+}$is the left ideal of $H$ generated by $\operatorname{ker}\left(\varepsilon_{K}\right)$ which is also a $H$-submodule coideal of $H$.

Theorem 6.6. Assume $B$ is a Hopf algebra with an invertible antipode and $X$ is a SaYD module. Assume also that $X$ has finite projective $B$-dimension. Now, let $0 \rightarrow K \rightarrow C \rightarrow C / K \rightarrow 0$ be a short exact sequence of $H$-counital $B$-module coalgebras such that both $C$ and $C / K$ are $B$-projective. Then two relative cyclic homologies we defined above for the pair $(C, K)$ with coefficients in $X$ are isomorphic.

Proof. Our definition dictates that the relative cyclic homology is defined through the short exact sequence

$$
0 \rightarrow \mathbb{C M}_{*}^{c}(K, C, X) \rightarrow \mathbb{C M}_{*}^{c}(C, B, X) \rightarrow \mathbb{C M}_{*}^{c}((C, K), B, X) \rightarrow 0
$$

However we also have the homotopy cofibration sequence

$$
\mathbb{C M}_{*}^{c}(K, B, X) \rightarrow \mathbb{C M}_{*}^{c}(C, B, X) \rightarrow \mathbb{C M}_{*}^{c}(C / K, B, X)
$$

meaning the two different relative theories we defined above are isomorphic.

Definition 6.7. A $k$-submodule $J$ of a bialgebra $B$ is called a bialgebra ideal if (i) $J$ is a two sided ideal of $B$ (ii) $J$ is a two sided coideal of $B$ (iii) $\varepsilon(J) \equiv 0$. If $B$ is a Hopf algebra then $J$ is called a Hopf ideal if $J$ is a bialgebra ideal and $S(J)=J$.

Theorem 6.8. Let $B$ be a bialgebra and $J$ be a bialgebra ideal of $B$. Assume also that $X$ is a stable $B$-module/comodule such that $z \cdot x=0$ for any $z \in J$. Then the relative cyclic homology of the pair $(B, J)$ with coefficients in $X$ is the same as the cyclic homology of the triple $(B / J, B / J, X)$.

Proof. Since $X$ is annihilated by $J$, the action of $B$ on $X$ actually splits as

$$
B \otimes X \stackrel{q_{B / J} \otimes i d_{X}}{\longrightarrow} B / J \otimes X \stackrel{\mu_{X}}{\longrightarrow} X
$$


where $B \stackrel{q_{B / J}}{\longrightarrow} B / J$ is the canonical quotient morphism. Thus $X$ is a stable $B / J$-module/comodule. Moreover,

$$
k \underset{B}{\otimes}\left((B / J)^{\otimes n+1} \otimes X\right)=k \underset{B / J}{\otimes}\left((B / J)^{\otimes n+1} \otimes X\right)
$$

for any $n \geq 0$ which implies

$$
\mathbb{C M}_{*}^{c}(B / J, B, X)=\mathbb{C M}_{*}^{c}(B / J, B / J, X)
$$

as we wanted to show.

Corollary 6.9. Let $B$ be a commutative Hopf algebra and $J$ be a Hopf ideal of B. Then the relative cyclic homology of the pair $(B, J)$ with coefficients in the 1-dimensional stable B-module/comodule $k$ is

$$
\mathrm{HC}_{n}^{\mathbb{C M}, c}((B, J), B, k) \cong \bigoplus_{i \geq 0} \mathrm{HH}_{n-2 i}(B / J, k)
$$

for any $n \geq 0$.

Proof. Theorem 6.8 implies $\mathbb{C M}_{*}^{c}(B / J, B, X) \cong \mathbb{C M}_{*}^{c}(B / J, B / J, X)$. Then Theorem 4.2 of [13] implies the result.

\section{THE DUAL THEORY}

In this section we will abstain from giving detailed proofs since they can be obtained from carefully dualizing corresponding proofs for the Hopf cyclic homology of module coalgebras given above.

7.1. Definitions. Recall that an an algebra $A$ is called a right $B$-comodule algebra if $A$ is a right $B$ comodule $A \stackrel{\rho_{A}}{\longrightarrow} A \otimes B$ and for any $a^{1}, a^{2} \in A$ we have

$$
\left(a^{1} a^{2}\right)_{(0)} \otimes\left(a^{1} a^{2}\right)_{(1)}=a_{(0)}^{1} a_{(0)}^{2} \otimes a_{(1)}^{1} a_{(1)}^{2}
$$

which is equivalent to saying $\rho_{A}$ is a morphism of algebras.

In [12] we constructed a cyclic theory $\operatorname{HC}_{*}^{\mathbb{C M}, a}(A, B, X)$ where $B$ is bialgebra, $A$ is a $B$-comodule algebra and $X$ is just a stable $B$-module/comodule. We also showed that in case $B$ is a Hopf algebra with an invertible antipode and $X$ is a stable anti-Yetter-Drinfeld module the theory we developed and the theory developed in [10] are the same. Here we develop a relative version of this dual theory in the bialgebra setting.

Definition 7.1. Given a $B$-comodule algebra $A$ and a $B$-subcomodule bilateral ideal $I \subset A$, we define the dual relative Hopf cyclic homology of the pair $(A, I)$ with coefficients in a stable $B$-module/comodule $X$ as the dual cyclic homology of the triple $(A / I, B, X)$. 
Definition 7.2. Given a $B$-comodule algebra $A$ and a $B$-subcomodule subalgebra $A^{\prime} \subset A$ we define the relative Hopf cyclic homology of $\left(A, A^{\prime}\right)$ with coefficients in a stable $B$-module/comodule $X$ as the homology of the complex

$$
\operatorname{coker}\left(\mathbb{C M}_{*}^{a}\left(A^{\prime}, B, X\right) \stackrel{\mathbb{C M}_{*}^{a}(i)}{\longrightarrow} \mathbb{C M}_{*}^{a}(A, B, X)\right)
$$

the cokernel of the canonical morphism of cyclic objects $\mathbb{C M}_{*}^{a}\left(A^{\prime}, B, X\right) \stackrel{\mathbb{C M}_{*}^{a}(i)}{\longrightarrow} \mathbb{C M}_{*}^{a}(A, B, X)$ coming from the inclusion $A^{\prime} \stackrel{i}{\longrightarrow} A$. This cyclic object is denoted by $\mathbb{C M}_{*}^{a}\left(\left(A, A^{\prime}\right), B, X\right)$.

Remark 7.3. Since any bilateral ideal is also a subalgebra, there are two possibly different definitions of dual relative cyclic homology. These different definitions will agree when the canonical morphism of cyclic objects $\mathbb{C M}_{*}^{a}((A, I), B, X) \rightarrow \mathbb{C M}_{*}^{a}(A / I, B, X)$ is a weak equivalence. This will happen if and only if we have excision in dual bialgebra cyclic homology.

\subsection{Cocommutative Hopf algebras.}

Theorem 7.4. Assume $K$ is a bialgebra ideal of $B$ such that the canonical epimorphism of coalgebras $B \stackrel{q}{\longrightarrow} B / K$ splits in the category of coalgebras. Assume also that $X$ is a stable $B$-module/comodule with the property that $\operatorname{Res}_{K}^{B}(X)=K \underset{B}{\square} X=0$. Then the dual relative cyclic homology of the pair $(B, K)$ with coefficients in $X$ is the same as the dual cyclic homology of the triple $(B / K, B / K, X)$.

Proof. Since $\operatorname{Res}_{K}^{B}(X)=0$ and $B \stackrel{q}{\longrightarrow} B / K$ splits, the $B$-coaction on $X$ splits as

$$
X \stackrel{\rho_{X}}{\longrightarrow} X \otimes B / K \rightarrow X \otimes B
$$

This means we have an equality

$$
\left((B / K)^{\otimes n+1} \otimes X\right) \underset{B}{\square} k=\left((B / K)^{\otimes n+1} \otimes X\right) \underset{B / K}{\square} k
$$

which means $\mathbb{C M}_{*}^{a}(B / K, B, X)=\mathbb{C M}_{*}^{a}(B / K, B / K, X)$. The result follows.

Corollary 7.5. Let $B$ be a cocommutative Hopf algebra and $K$ be a Hopf ideal of $B$ such that the epimorphism $B \stackrel{q}{\longrightarrow} B / K$ splits as a morphism of coalgebras. Then one has

$$
\mathrm{HC}_{n}^{\mathbb{C M}, a}\left(B / K, B, k_{(1, \varepsilon)}\right) \cong \bigoplus_{i \geq 0} \mathrm{HH}_{n-2 i}(B / K, k)
$$

Proof. Theorem [7.4 above and Theorem 4.1 of [13].

Example 7.6. Let $G$ be an arbitrary discrete group and $H$ be a normal subgroup of $G$. Consider the two sided ideal $a_{H}$ of $k[G]$ generated by elements of the form $(h-1)$ where $h \in H$. First, observe that $a_{H}$ is also a two sided coideal of the Hopf algebra $k[G]$ and is stable under the antipode map $S(g)=g^{-1}$. Next observe 
that $k[G] / a_{H} \cong k[G] \underset{H}{\otimes} k \cong k[G / H]$ and $a_{H} \underset{G}{\square} k=0$. Now for every coset $g H$ in $G / H$ pick a representative $s(g H) \in G$. The induced map $k[G / H] \stackrel{s}{\longrightarrow} k[G]$ is not a morphism of algebras but is a morphism of coalgebras since $\Delta(x)=(x \otimes x)$ for any $x$ from $k[G]$. So, the canonical quotient $k[G] \rightarrow k[G / H]$ splits as a morphism of coalgebras. Therefore the relative Hopf cyclic homology of $k[G]$ with respect to the Hopf ideal $a_{H}$ with coefficients in the trivial $k[G]$-module/comodule $k$ is given by

$$
H C_{n}^{\mathbb{C M}, a}\left(k[G] / a_{H}, k[G] ; k\right) \cong \bigoplus_{i \geq 0} H H_{n-2 i}(k[G / H], k) \cong \bigoplus_{i \geq 0} H_{n-2 i}(G / H, k)
$$

\subsection{Excision in dual Hopf cyclic homology.}

Theorem 7.7 (Excision in Dual Hopf Cyclic Homology). Let B be a Hopf algebra with an invertible antipode and $X$ be a stable anti-Yetter-Drinfeld module. Assume $A$ is a unital B-comodule algebra and $I$ is a $H_{-}$ unital B-subcomodule ideal of $A$. Assume also that both $I$ and $A$ are $B$-co-projective. Then the short exact sequence of $B$-comodule algebras $0 \rightarrow I \rightarrow A \rightarrow A / I \rightarrow 0$ yields a homotopy cofibration sequence

$$
\mathbb{C M}_{*}^{a}(I, B, X) \rightarrow \mathbb{C M}_{*}^{a}(A, B, X) \rightarrow \mathbb{C M}_{*}^{a}(A / I, B, X)
$$

Remark 7.8. In this version of the excision, we do not need any condition on the $B$-co-projective dimension of $X$ since the resulting spectral sequence computing the hyper Cotor functors is convergent even when $X$ does not have finite co-projective dimension.

Remark 7.9. If we drop $B$, in other words if we set $B=k$, then Theorem[7.7 reduces to Wodzicki's excision theorem [17. Again, we suspect that we have a Cuntz-Quillen type excision [7] in periodic equivariant cyclic homology, if we drop the H-unitality condition.

Conjecture 7.10. Let $B$ be a Hopf algebra with an invertible antipode and $X$ be a SaYD module. Let $0 \longrightarrow J \longrightarrow A \longrightarrow A / J \longrightarrow 0$ be a sequence of $B$-comodule algebras such that both $J$ and $A$ are $B$-coprojective. Then one has a 6-term exact sequence in periodic cyclic homology of the form

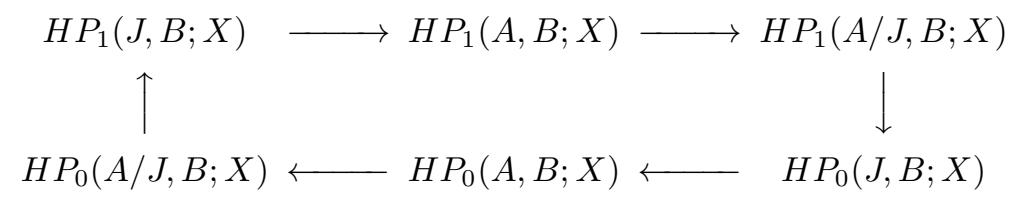


7.4. Integrals. Recall that a linear functional $B \stackrel{\eta}{\longrightarrow} k$ is called a normalized left integral if

$$
b_{(1)} \eta\left(b_{(2)}\right)=\eta(b) \mathbb{I} \quad \text { and } \quad \eta(\mathbb{I})=1
$$

for any $b \in B$. Right integrals are defined similarly.

Let $B$ be a Hopf algebra with an invertible antipode. Then it is easy to see that $B$ admits a normalized left integral iff $B$ admits a normalized right integral. The following theorem is standard (cf. e.g. [16] for a proof):

Proposition 7.11. Assume $B$ is a Hopf algebra with an invertible antipode. $B$ admits a left integral iff $B$ is co-semi-simple as a coalgebra.

Corollary 7.12. Let $B$ be a Hopf algebra with an invertible antipode and $X$ be a SaYD module. Assume $B$ admits a left integral. Let $A$ be a unital B-comodule algebra and I be a $H$-unital B-submodule ideal of

A. Then there is a homotopy cofibration sequence given in Equation 7.2

Proof. Existence of left integral implies $B$ is co-semi-simple which in turn implies every $B$-comodule is $B$-co-projective. The result follows.

\section{REFERENCES}

[1] R. Akbarpour and M. Khalkhali. Hopf algebra equivariant cyclic homology and cyclic homology of crossed product algebras. J. Reine Angew. Math., 559, 2003.

[2] A. Connes. Noncommutative differential geometry. Inst. Hautes Études Sci. Publ. Math., (62):257-360, 1985.

[3] A. Connes and H. Moscovici. Hopf algebras, cyclic cohomology and transverse index theorem. Comm. Math. Phys., 198:199-246, 1998.

[4] A. Connes and H. Moscovici. Cyclic cohomology and Hopf algebras. Lett. Math. Phys., 48(1):97-108, 1999.

[5] A. Connes and H. Moscovici. Cyclic cohomology and Hopf algebra symmetry. Lett. Math. Phys., 52(1):1-28, 2000.

[6] A. Connes and H. Moscovici. Background independent geometry and Hopf cyclic cohomology. Preprint at arXiv:math.QA/0505475 May 2005.

[7] J. Cuntz and D. Quillen. Excision in bivariant periodic cyclic cohomology. Invent. Math., 127(1):67-98, 1997.

[8] Y. Doi. Homological coalgebra. J. Math. Soc. Japan, 33:31-50, 1981.

[9] M. A. Farinati and A. Solotar. Cyclic cohomology of coalgebras, coderivations and De Rham cohomology. In Hopf Algebras and Quantum Groups, volume 209 of Lecture Notes in Pure and Applied Mathematics, pages 105-129. Marcel Dekker Inc., 2000.

[10] P. M. Hajac, M. Khalkhali, B. Rangipour, and Y. Sommerhäuser. Hopf-cyclic homology and cohomology with coefficients. C. R. Math. Acad. Sci. Paris, 338(9):667-672, 2004.

[11] P. M. Hajac, M. Khalkhali, B. Rangipour, and Y. Sommerhäuser. Stable anti-Yetter-Drinfeld modules. C. R. Math Acad. Sci. Paris, 338(8):587-590, 2004.

[12] A. Kaygun. Bialgebra cyclic homology with coefficients. K-Theory, 34(2):151-194, February 2005. 
[13] M. Khalkhali and B. Rangipour. A new cyclic module for Hopf algebras. K-Theory, 27(2):111-131, 2002.

[14] M. Khalkhali and B. Rangipour. Invariant cyclic homology. K-theory, 28(2):183-205, 2003.

[15] M. Khalkhali and B. Rangipour. Introduction to Hopf-cyclic cohomology (to appear in Proc. Max Planck Inst. Conf. Noncommutative Geometry and Number Theory). Preprint at arXiv:math.QA/0503244 March 2005.

[16] M. E. Sweedler. Hopf Algebras. W. A. Benjamin, Inc., 1969.

[17] M. Wodzicki. Excision in cyclic homology and in rational algebraic K-theory. Ann. of Math., 129(3):591-639, 1989.

E-mail address: akaygun@uwo.ca

E-mail address: masoud@uwo.ca

Department of Mathematics, The University of Western Ontario, London, Ontario n6A 5B7, Canada 